\title{
Injury outcome among helmeted and non-helmeted motorcycle riders and passengers at a tertiary care hospital in north-western Tanzania
}

PHILLIPO L. CHALYA ${ }^{\prime *}$, ISIDORI H. NGAYOMELA ${ }^{2}$, JOSEPH B. MABULA ${ }^{1}$, NKINDA MBELENGE ${ }^{2}$, RAMESH M. DASS ${ }^{2}$, ALPHONCE CHANDIKA', JAPHET M. GILYOMA', ANTHONY KAPESA ${ }^{3}$ and SOSPATRO E. NGALLABA ${ }^{3}$

${ }^{1}$ Department of Surgery, Catholic University of Health and Allied Sciences-Bugando, Mwanza, Tanzania

${ }^{2}$ Department of Orthopaedics \& Traumatology, Catholic University of Health and Allied Sciences-Bugando, Mwanza, Tanzania

${ }^{3}$ Department of Community Medicine, Catholic University of Health and Allied Sciences-Bugando, Mwanza, Tanzania

\begin{abstract}
Motorcycle helmets have been reported to reduce the risk of death and head injuries following motorcycle accidents. The aim of this descriptive prospective study was to determine the injury outcome among helmeted and non-helmeted motorcyclists and passengers at a tertiary hospital in north-western Tanzania. A total of 654 patients involved in the motorcycle accident were studied. Of these, $468(71.6 \%)$ were motorcyclists (riders) and the remaining $186(28.4 \%)$ were passengers. The median age of patients at presentation was 26 years. Male outnumbered females by a ratio of $4.5: 1$. Helmet use was reported in 312 (47.7\%) patients. Non-helmeted patients were young compared with helmeted patients and this was statistically significant $(p=0.021)$. The rate of helmet use was significantly higher among motorcyclists than among passengers $(p=0.004)$. History of alcohol consumption prior to the accident was reported in 212 (32.4\%) patients. The rate of helmet use was significantly low among alcohol consumers compared with non-alcohol consumers $(p=0.011)$. Lack of helmet use was significantly associated with abnormal head Computed Tomography scans, admission to the Intensive care unit, severe trauma, and worse traumatic brain injury severity $(p<0.001)$. Helmet use was significantly associated with shorter period of hospitalization and reduced mortality rate $(p<0.001)$. Motorcycle helmet use is still low in this part of Tanzania and this poses a great impact on injury outcome among motorcycle injury patients. This observation calls for action to implement more widespread injury prevention and helmet safety education and advocacy.
\end{abstract}

Keywords: motorcycle accidents, helmet use, injury outcome, Tanzania

\section{Introduction}

Road traffic injuries are a major cause of death and disability globally, with a disproportionate number occurring in developing countries (Nantulya \& Reich, 2002; Peden, 2004; Ameratunga et al., 2006). The increase in road traffic injuries in most developing countries has been attributed to by the escalating use of motorcycles for commercial transportation of commuters, goods, and services (Solagberu et al., 2006; Galukande et al., 2009; Dee, 2009; Chalya et al., 2010). Motorcycle users are vulnerable on the road and are at high risk of death resulting from head and neck injuries (Solagberu et al., 2006; Chalya et al., 2010). One way of protecting the riders and passengers from these injuries, in the event of an accident, is the use of helmets. There is a lot of documentation on the safety benefits of helmets usage when riding motorcycles which includes a reduction in the number of recorded deaths, shorter period of hospitalization and a reduction in the medical costs incurred by riders (Brandt et al., 2002; Liu et al., 2008).

Mandatory helmet legislation is one way of protecting riders of motorcycles in many countries (Grima et al., 1995; Liu et al., 2008). Researchers have shown that helmet legislation compels riders to use helmets, which ultimately leads to reduction of deaths resulting from motorcycle accidents (Grima et al., 1995; Liu et al., 2008). A study in the United States indicated that the average motorcyclist death rates were reduced by $22-33 \%$ in certain states with universal helmet legislation when compared with an era of no helmet legislation. It was also indicated that

\footnotetext{
* Correspondence: Phillipo L. Chalya; E-mail: drphillipoleo@yahoo.com
} 
helmet legislation that covered only certain categories of riders reduced the average death rates by $7-10 \%$ (Corad et al., 1996).

In developing countries such as Tanzania, motorcycle use is very common and is commonly used for commercial transportation. This is because it has some strong advantages such as its easy manoeuvrability, superior fuel economy and time saving over the commercial car taxis. However, the use of motorcycles as a means of commercial transport in Tanzania and other developing countries is characterized by non-helmet use by riders and their passengers, passenger overload, lack of certified driver training and valid licensing, over speed and reckless driving, poor regulation and law enforcement and possible use of alcohol and drugs (Museru \& Leshabari, 2002; Chalya et al., 2010). In addition, in Tanzania, there is less documentation on helmet usage by both riders and passengers as a safety measure.

Motorcycle helmet use has been reported to be an effective way to reduce death and disability after traffic collisions, and enactment of universal helmet laws has been suggested as a means to enforce helmet use (Offner et al., 1992; Brandt et al., 2002; Keng, 2005). However, studies have shown that helmet use in developing countries is low (Odelowo, 1994; Oluwadiya et al., 2004). Therefore safety measures should be strong to prevent a lot of deaths from motorcycle riding. It was observed by the researchers that the number of motorcycles being involved in accident is on the ascendancy and involves both male and female. Most of these accidents witnessed involved victims without helmets. One study indicated that motorcycle riders in developing countries seemed more likely to ignore safety measures (Oluwadiya et al., 2004). Therefore, the aim of this study was to determine the injury outcome among helmeted and non-helmeted motorcyclists and passengers at Bugando Medical Centre, a tertiary hospital in north-western Tanzania.

\section{Materials and Methods}

\section{Study site and design}

This descriptive prospective study was conducted at Bugando Medical Centre (BMC) in Mwanza, north-western Tanzania. BMC, located along the shore of Lake Victoria has a 1000 bed capacity and serves as a teaching hospital for the Catholic University of Health and Allied SciencesBugando and other paramedics. It is one of the four largest referral hospitals in the country and serves as a referral centre for tertiary specialist care for a catchment population of approximately 13 million people from neighbouring regions. The hospital receives all trauma patients from the city and from neighbouring regions as there is no trauma centre or established advanced prehospital care in Mwanza city. The study was conducted from January to December 2013 at the Accident and Emergency (A \& E) Department of Bugando Medical Centre.

\section{Study population}

The study included all motorcycle injury patients who presented to the $A \& E$ department during the study period and thereafter admitted to the trauma wards of Bugando Medical Centre. Patients who failed to give proper information about the injury and those who had no relative to consent for the study were excluded from the study. Recruitment of patients to participate in the study was done at the A \& E Department. Patients were screened for inclusion criteria and those who met the inclusion criteria were consecutively enrolled into the study.

Patients with severe injuries were first resuscitated in the A\&E department according to Advanced Trauma Life Support (ATLS). From the A \& E department, patients were taken into the surgical or orthopaedic and trauma wards from where necessary investigations were completed and further treatment was instituted. Patients with severe injuries requiring ventilatory support were admitted in the intensive care unit (ICU).

Patients requiring surgical treatment were prepared for emergency surgery. The severity of injury was determined using the Kampala trauma score II (KTS II) (Mutooro et al., 2010). KTS II 
is scored based on the following parameters (A) age in years (i.e. 5-55 =1, $<5$ and $>55=0$ ), (B) number of serious injuries (i.e. none $=2$, one injury $=1$ and $>$ one injury $=0$ ), (C) systolic blood pressure (i.e. $>89 \mathrm{mmHg}=2,89-50 \mathrm{mmHg}=1, \leq 49 \mathrm{mmHg}$ ), (D) respiratory rate (i.e. $0-29 / \mathrm{min}=2$, $>30 / \mathrm{min}=1$ and $\leq 29 / \mathrm{min}$ ) and neurologic status (i.e. alert = 3, responds to verbal stimuli $=2$, responds to painful stimuli $=1$ and unresponsive $=0$ ) on presentation. Kampala Trauma Score total $=A+B+C+D+E$. Severe injury consists of a KTS II $\leq 6$, moderate injury 7-8, and mild injury 9-10. Patients with head injuries were classified according to Glasgow Coma Scale (GCS) into: severe (GCS 3-8), moderate (GCS 9-12) and mild (GCS 13-15). An initial systolic blood pressure (SBP) from each patient was also recorded on admission to assess the presence of shock. Routine investigations including haematological (haemoglobin, blood grouping \& cross-matching), biochemical (serum creatinine and serum electrolytes) and imaging (plain x-rays of the chest $/$ abdomen and limbs, abdominal ultrasound and CT scan) were performed on admission.

Depending on the type of injury, patients were treated either conservatively or surgically. Data were collected using a pretested questionnaire. Included in the questionnaire were sociodemographic data (age, sex, education and occupation), mechanism of injury, helmet use at the time of accident, alcohol consumption prior to accident, injury-arrival interval, admission pattern, type and pattern of injury, trauma scores (KTS II \& GCS), body region injured, treatment offered, complications of treatment. Outcome variables were length of hospital stay, mortality and disability.

\section{Data analysis}

The statistical analysis was performed using statistical package for social sciences (SPSS) version 17.0 for Windows (SPSS, Chicago IL, USA). The median (+ IQR) and ranges were calculated for continuous variables whereas proportions and frequency tables were used to summarize categorical variables. Chi-square $\left(\chi^{2}\right)$ test were used to test for the significance of association between the independent (predictor) variables (socio-demographic data (age, sex, education and occupation), mechanism of injury, helmet use at the time of accident, alcohol consumption prior to accident, injury-arrival interval, admission, type and pattern of injury, trauma scores, body region injured, treatment offered, complications of treatment.) and dependent (outcome) variables (treatment complications, hospital stay, mortality and disability) in the categorical variables. The level of significance was considered as $\mathrm{p}$-value $<0.05$. Multivariate logistic regression analysis was used to determine predictor variables that predict the treatment complications, hospital stay, mortality and disability

\section{Ethical considerations}

Ethical approval to conduct the study was obtained from the CUHAS-Bugando/BMC joint institutional ethic review committee before the commencement of the study. Informed consent was sought from each patient before being enrolled into the study.

\section{Results}

Out of 987 roar traffic injuries who presented to our centre during the study period, $654(66.3 \%)$ were related to motorcycle injuries affecting mainly the motorcyclists and passengers. Pedestrians were not included in this study. The age of patients at presentation ranged from 9 to 76 years with a median of 26 years (+ IQR of 24 to 28 years). The modal age group was 21-30 years accounting for $348(53.2 \%)$ patients. Five hundred thirty four $(81.7 \%)$ were males and 120 (18.3\%) females with a male to female ratio of $4.5: 1$. Amongst the 654 patients, 468 (71.6\%) were motorcyclists (riders) and the remaining 186 (28.4\%) were passengers. All riders were males. The educational level of victims revealed that of the 468 motorcyclists, $402(85.9 \%)$ had primary level of education or none at all. 112 (60.2\%) of passengers had at least secondary education with 23 (12.4\%) having tertiary education. Findings from this study revealed that $422(64.5 \%)$ of the victims 
were commercial motorcyclists, 152 (23.2\%) were students, 62 (9.5\%) were farmers and 18 (2.8\%) were public servants. Five hundred and fifty six (85.0\%) patients had no identifiable health insurance.

Motorcycle versus vehicle accounted for the majority of types of collision in $422(64.5 \%)$ patients. This was followed by lone versus motorcyclists collision, motorcycle versus motorcycle and other collisions in 118 (18.0\%), 102 (15.6\%) and 12 (1.8\%) patients respectively. The lone motorcyclist crashes were collisions with stationary objects and unexpected loss of control.

Helmet use was reported in $312(47.7 \%)$ patients. The median age of helmeted patients at presentation was 29 years (+ IQR of 25 to 32 years), while it was 22 years (+ IQR of 20 to 26 years) for non-helmeted patients. The difference in age distribution of helmeted and nonhelmeted patients was statistically significant in multivariate logistic regression analysis ( $p=$ 0.021). The rate of helmet use was significantly higher among motorcyclists (55.6\% i.e. $260 / 468$ ) than among passengers $(28.0 \%$ i.e. $52 / 186)(p=0.004)$. History of alcohol consumption prior to the accident was reported in $212(32.4 \%)$ patients. The rate of helmet use was low among alcohol consumers ( $37.7 \%$ i.e. $80 / 212)$ as compared to non-alcohol consumers $(52.5 \%$ i.e. $232 / 442)$ and this was statistically significant in multivariate logistic regression analysis $(p=0.011)$. The vast majority of patients $(486,74.3 \%)$ reported to the A \& E department within 24 hours after injury and most of them were attended to within 4-6 hours of arrival. According to multivariate logistic regression analysis, there was no statistically significant association between helmet use and injury-arrival time $(\mathrm{p}=0.145)$.

Musculoskeletal (extremities) and the head were the most common body region injured affecting $66.4 \%$ and $54.7 \%$ of patients, respectively (Table 1). In patients who had head injuries, the Glasgow coma scale indicated that 140 (39.1\%) patients sustained moderate head injury, 116 (32.4\%) patients sustained mild head injury and 102 (28.5\%) patients had severe head injury. No patient who had motorcycle helmet at the time of accident sustained severe head injury. There was no association between helmet use and occurrence of musculoskeletal injuries in multivariate logistic regression analysis $(p=0.987)$.

Table 1: Distribution of patients according to body region injured

\begin{tabular}{lll}
\hline Body region injured & Frequency & Percentage \\
\hline Musculoskeletal & 434 & 66.4 \\
Head/neck & 358 & 54.7 \\
Abdominal & 112 & 17.1 \\
Chest & 94 & 14.4 \\
Spines & 12 & 1.8 \\
Pelvis & 9 & 1.4 \\
Genitalia & 7 & 1.1 \\
\hline
\end{tabular}

According to Kampala Trauma Score II (KTS II), the majority of patients sustained moderate trauma (KTS II = 7-8) in 370 (56.6\%). Severe trauma (KTS II $\leq 6)$ and mild trauma (KTS II = 9-10) were recorded in $268(41.0 \%)$ and 16 (2.4\%) patients respectively. Only 34 (12.7\% i.e. 34/268) of helmeted patients sustained severe trauma as compared to non-helmed patients $(72.0 \%$ i.e. $278 / 386)$ and this was statistically significant in multivariate logistic regression analysis ( $p=$ $0.001)$.

Isolated injuries occurred in 435 (66.5\%) patients while 219 (33.5\%) patients had multiple injuries. There was no statistically significant association between helmet use and the number of injuries $(p=0.439)$. Open wounds (i.e. bruises, abrasions, lacerations, crush wounds, traumatic amputation, etc) and fractures were the most common type of injuries sustained (Table 2). According to multivariate logistic regression analysis, there was no statistically significant association between helmet use and the type of injuries sustained $(p=0.876)$. 
Table 2: Distribution of patients according to the type of injuries

\begin{tabular}{lll}
\hline Type of injury & Frequency & Percentage \\
\hline Open wounds & 345 & 52.8 \\
Fractures & 159 & 24.3 \\
Intracranial haemorrhage & 74 & 11.3 \\
Visceral injuries & 48 & 7.3 \\
Traumatic limb amputation & 15 & 2.3 \\
Pneumothorax & 9 & 1.4 \\
Other injuries & 7 & 1.1 \\
\hline
\end{tabular}

In this study, CT scan of the brain and skull was requested in $242(67.6 \%)$ patients, but it was only performed in $76(21.2 \%)$ patients due to financial problems and other logistics. Out of 76 patients who had CT scan done, abnormal findings were detected in 68 (89.4\%) patients (Table 3). The rate of abnormal CT scan findings was significantly high among non-helmeted patients compared to helmeted patients in multivariate logistic regression analysis $(p=0.023)$.

Table 3: CT scan of the brain findings ( $\mathrm{N}=68)$

\begin{tabular}{lll}
\hline CT Scan abnormality & Frequency & Percentage \\
\hline Scalp hematoma & 45 & 66.2 \\
Generalized cerebral oedema & 40 & 58.8 \\
Skull fractures & 34 & 50.0 \\
Epidural hematoma & 12 & 17.6 \\
Subdural hematoma & 8 & 11.8 \\
Pneumocephalus & 5 & 7.4 \\
Intracerebral hematoma & 5 & 7.4 \\
Subarachnoid hematoma & 3 & 4.4 \\
\hline
\end{tabular}

The majority of patients, $502(76.8 \%)$ were admitted in the general surgical wards. $12(1.8 \%)$ patients had an overnight stay at the Accident \& Emergency department and then discharged. 140 (21.4\%) patients were admitted in the intensive care unit (ICU); of these, $104(74.3 \%)$ necessitated ventilatory support. The majority of patients, $124(88.6 \%)$ who were admitted in the ICU were not helmeted. There was a statistically significant association between helmet use and the need for ICU admissions ( $p=0.012$ ). The majority of patients, 598(91.4\%) were treated surgically. Wound debridement was the most common procedure performed in $56.9 \%$ of patients (Table 4).

Table 4: Distribution of patients according to the type of surgical procedure performed $(\mathrm{N}=598)$

\begin{tabular}{lll} 
Type of surgical procedure & Frequency & Percentage \\
\hline Wound debridement & 340 & 56.9 \\
Treatment of fractures & 159 & 26.6 \\
Burr holes/craniotomy & 56 & 9.4 \\
Exploratory laparotomy & 48 & 8.0 \\
Skin grafting/flaps & 34 & 5.7 \\
Limb amputation & 18 & 3.0 \\
Underwater seal drainage & 14 & 2.3 \\
Suprapubic catheterization & 11 & 1.8 \\
Scrotoplasty & 5 & 0.8 \\
\hline
\end{tabular}

A total of 184 complications were recorded in 132 patients giving a complication rate of $20.2 \%$. Of these, wound sepsis was the most common complication accounting for $30.4 \%$ of cases (Table 5). 
There was no statistically significant association between helmet use and the presence of complications in multivariate logistic regression analysis $(p=0.679)$.

Table 5: Distribution of patients according to complications $(\mathrm{N}=184)$

\begin{tabular}{lll}
\hline Complication & Frequency & Percentage \\
\hline Wound sepsis & 56 & 30.4 \\
Complications of fractures & 23 & 12.5 \\
Complications of abdominal surgery & 12 & 6.5 \\
Skin grafting failure & 8 & 4.3 \\
Re-amputation & 7 & 3.8 \\
Tetanus & 7 & 3.8 \\
Emphysema thoracic & 4 & 2.2 \\
Neurological deficit & 3 & 1.6 \\
Subcutaneous emphysema & 3 & 1.6 \\
\hline
\end{tabular}

The overall length of hospital stay (LOS) ranged from 1 to 124 days with a median of 28 days (+IQR of 26 to 32 days). The median LOS for non-survivors was 6 days (range 1-10 days). The length of ICU stay ranged from 1 day to 36 days with a median of 12 days (+IQR of 8 to 16 days). The overall median length of hospital stay was long among non-helmeted patients ( 38 days) compared with helmeted patients ( 24 days) and according to multivariate logistic regression analysis, this was statistically significant $(p=0.022)$.

In this study, one hundred thirty-five patients died, giving a mortality rate of $20.6 \%$. Mortality rate was high among non-helmeted patients (84.4\% i.e. 114 deaths) compared with helmeted patients (15.6\% i.e. 21 deaths). This difference was statistically significant in multivariate logistic regression analysis $(p=0.001)$. Of the 519 survivors, $428(82.5 \%)$ had good recovery and were discharged well. Sixty-six (12.7\%) were discharged with permanent disabilities. Fifteen $(2.9 \%)$ patients were absconded, teen (1.9\%) were discharged against medical advice. The rate of permanent disability was slightly high among non-helmeted patients ( $56.1 \%$ i.e. 37 patients) compared with helmeted patients (43.9\% i.e. 29 patients). However, this difference was not statistically significant in multivariate logistic regression analysis $(p=0.345)$.

\section{Discussion}

Motorcycle injuries constitute a major public health problem in developing countries and are a common cause of road traffic injuries (Nantulya \&Reich, 2002; Peden, 2004; Ameratunga et al., 2006; Dee, 2009). In this study, motorcycle related injuries occurred in $66.3 \%$ of all road traffic injuries, a figure which is higher than that reported previously at the same centre (Chalya et al., 2010, 2012, 2013) reflecting increase in the magnitude of the problem in our setting. Motorcycle use is becoming popular in Tanzania as it has become a cheaper and easier means of transportation in most cities. However its use is characterized by low level of use of safety equipment such as helmet by both the riders and their passengers contributing significantly to high risk of death and head injuries (Chalya et al., 2010).

Studies have shown that the majority of commercial motorcyclists have low level of education and this could be partly attributed by the fact that those who are less educated found themselves with few chances of getting other jobs and thus engage into commercial motorcycling business as a last resort following unemployment regardless of being a risky job (Iribhogbe \& Odai, 2009). This observation is reflected in our study where most of riders had either primary or no formal education and the majority of them were unemployed. This observation has an implication on the awareness of the factors contributing to crush occurrence and injury severity related to motorcycle accidents. 
The majority of patients in the present study were young in their second decade of life which is in keeping with the known peak occurrence of motorcycle injuries in most studies (Chalya et al., 2010; Nwadiaro et al, 2011). This is the active age group likely to be involved in highrisk activities such as recklessness driving/riding, over-speeding, driving/riding under the influence of alcohol and driving/riding without wearing any protective gears. The male preponderance in this study is in agreement with several other reports (Solagberu et al., 2006; Chalya et al., 2010; Nwadiaro et al, 2011). It is observed that nearly all commercial motorcyclists are males and riders constituted the single largest risk group (71.6\%). This agrees with other studies which identify riders as the majority of motorcycle crash victims presenting to hospitals (Solagberu et al., 2006; Chalya et al., 2010).

Motorcycle helmets have been reported in literature to reduce the risk of death and head injuries (Offner et al., 1992; Brandt et al., 2002; Keng, 2005). However, studies have shown that helmet use in developing countries is low (Odelowo, 1994; Oluwadiya et al., 2004). In the present study, helmet use was recorded in $47.7 \%$ which is higher than that reported previously at the same centre (Chalya et al., 2010, 2012, 2013) and in Uganda by Galukande et al (2009), but low compared to what was reported by Catherine et al. (2008) in Victoria. These differences in the rate of helmet use reflect differences in awareness of factors contributing to crush occurrence and injury severity related to motorcycle accidents between these countries and poor enforcement of traffic laws. The differences in helmet use may also imply different attitudes to helmet wearing between these countries.

In accordance to other studies (Rowland et al., 1996; Corad et al., 1996; Byrnes \& Gerberich, 2012), non-helmeted patients in this study were found to be young as compared to helmeted patients. The reason behind not wearing helmet among those in the young age may be due to the fact that young people generally tend to adopt risky behaviour and attitudes as compared to their elder counterparts (Seleye-Fubara \& Ekere, 2003).

In this study, a history of alcohol consumption prior to the accident was reported in $32.4 \%$ of cases, a figure which is higher than that reported previously by others (Sood, 1988; Oluwadiya et al., 2004; Chalya et al., 2010, 2012). The rate of helmet use in the present study was significantly low among alcoholic patients as compared to non-alcoholic patients. The role of alcohol in impairing driving ability is well documented. Alcohol usage causes carelessness and loss of concentration as well as over speeding and neglecting the use of safety equipment such as helmet.

As reported in previous studies (Oluwadiya et al., 2004; Chalya et al., 2010, 2012), findings in the present study found that musculoskeletal and head injuries were the most common body region injured attributing the latter to the low use of motorcycle helmets in our study; a situation seen in other developing countries. In the present study, no patient who was helmeted at the time of injury sustained head injury reflecting its importance in prevention of head injuries among motorcycle injury patients. Several studies have reported on the effectiveness of helmet in protecting against head injury (Julian et al., 2002; Chang \& Yeh, 2007; Hung et al., 2008; Brown et al., 2009; Lin et al., 2003). Motorcycle helmets reduce the risk of head injury in motorcycle riders and passengers who crash (WHO, 2006). Helmets create an additional layer for the head and thus protect the wearer from some of the more severe forms of traumatic brain injury. In spite of the protective nature of helmets, and the impact of traumatic brain injury for motorcycle users (WHO, 2006), low rate of helmet use in middle and low-income countries is the commonly observed phenomena (Odelowo, 1994; Oluwadiya et al., 2004). To make matters worse, in spite of the low rate of helmet use, helmets are not worn properly by having the chin strap fastened (Liu et al., 2008). Lack of association between helmet use and occurrence of musculoskeletal injuries in the present study reflects failure of motorcycle helmets to reduce the risk of musculoskeletal injuries among motorcycle riders and passengers.

The finding that lack of helmet use in the present study was significantly associated with abnormal head/ brain CT scans agrees with a recent study in the United States (Liu et al., 2008). In 
this study, the rate of abnormal head/brain CT scan findings was significantly high among nonhelmeted patients compared to helmeted patients. The reason for the high rate of abnormal CT scan findings among non-helmeted patients may be attributed to the fact that motorcycle riders or passengers who do not wear a helmet run a much higher risk of sustaining head and traumatic brain injuries, or a combination of them resulting into abnormal head/ brain CT scan findings.

More than eighty percent of patients who were admitted in the ICU in our series were not helmeted which is in keeping with other studies done elsewhere (Liu et al., 2008; Byrnes \& Gerberich, 2012). In the present study, the use of motorcycle helmet was statistically significant associated with the need for ICU admissions. The need for ICU admissions among non-helmeted patients can be explained by the fact that the majority of non-helmed patients sustained severe trauma and severe head injuries necessitating them to be admitted in the ICU.

In keeping with other studies (Byrnes \& Gerberich., 2012), the overall length of hospital stay in our study was significantly longer in patients who did not use helmets compared with those who used helmets. Prolonged length of hospital stay among non-helmeted patients in our study may be attributed to the fact that the majority of motorcycle riders and passengers who did not wear a helmet sustained severe trauma and severe traumatic brain injuries that predisposed them to prolonged hospitalization.

The use of motorcycle helmets has been reported to decrease the overall death rate of motorcycle crashes when compared with non-helmeted riders (Offner et al., 1992; Brandt et al., 2002; Keng, 2005; Liu et al., 2008). In the current study, the overall mortality rate was $20.6 \%$, but it was significantly high $(84.4 \%)$ in patients who did not use helmets compared to those who used helmets reflecting the importance of motorcycle helmets in prevention of deaths among motorcycle injury patients. High mortality rate among non-helmeted patients in our study can be explained by the fact that most of non-helmeted patients sustained severe trauma and severe head injuries which were found to be predictors of deaths in the current study. In this study, the rate of permanent disability was slightly high among non-helmeted patients as compared to helmeted ones, but this association was found to be not statistically significant. The lack of association between helmet use and permanent disability may be attributed to the fact that the majority of permanent disabilities in this study were related to musculoskeletal injuries for which motorcycle helmets have no protective role.

The potential limitation of this study is the fact that the rate of motorcycle helmet use may not be the true estimate and does not reflect the true picture in Mwanza city. This is due to unreported patients, patients who died at scene or who did not reach our hospital because of treatment of minor injuries in private hospitals. Also information regarding motorcycle helmet use and alcohol consumption was based on self-reporting which may not be reliable. This might have introduced some bias in our findings. A better picture of the magnitude of the problem in our setting requires comprehensive data to include all hospitals in Mwanza city. However, despite these limitations, the study has provided local data that can be utilized by health care providers to plan for preventive strategies as well as establishment of management guidelines for patients with motorcycle injuries.

In conclusion, motorcycle helmet use among riders and passenger is generally low in this part of Tanzania. Lack of helmet use was significantly associated with abnormal head CT scans, admission to the ICU, severe trauma, and worse traumatic brain injury severity. Also, helmet use was significantly associated with short period of hospitalization and reduced mortality rate. Therefore, there is a need for public awareness campaigns on the safety benefits of helmets to increase its prevalence in this region. The education on helmet use must be accompanied by sustained enforcement of the road traffic law by the traffic police to ensure compliance and change in attitudes. 


\section{Acknowledgements}

The authors gratefully acknowledge the contributions of the entire staff of the surgical department of the study centre and all the Senior House Officers that assisted with data collection.

\section{References}

Ameratunga, S., Hijar, M. \& Norton, R. (2006) Road traffic injuries: confronting disparities to address a global health problem. Lancet 367, 1533-1540.

Brandt, M.M., Ahrns, K.S., Corpron, C.A., Franklin, G.A. \& Wahl, W.L. (2002) Hospital cost is reduced by motorcycle helmet use. Journal of Trauma 53, 469-4671.

Brown, V., Hejl, K., Bui, E., Tips, G. \& Coopwood, B. (2009) Risk factors for riding and crashing a motorcycle unhelmeted. Journal of Emergency Medicine 41, 441-446.

Byrnes, M. \& Gerberich, S. (2012) Motorcycle helmet use and legislation: a systematic review of the literature. Minnesota Medicine 95, 60-65.

Catherine, A.B., Franz, E.B., Penny, B. \& Lisa, N.S. (2008) The increasing problem of motorcycle in Children and Adolescents. Medical Journal of Australia 189, 17-20.

Chalya, P.L., Dass, R.M., Mchembe, M.D., Mbelenge N., Ngayomela, I.H., Chandika, A.B,. Gilyoma, J.M., Mabula, J.B. (2013) City wide trauma experience in Mwanza, Tanzania: a need for urgent intervention. Journal of Trauma Management \& Outcomes 7, 9.

Chalya, P.L., Mabula, J.B., Dass, R.M., Mbelenge, N., Ngayomela, I.H., Chandika, A.B. \& Gilyoma, J.M. (2012) Injury characteristics and outcome of road traffic crash victims at Bugando Medical Centre in Northwestern. Tanzania. Journal of Trauma and Management Outcome, 6:1.

Chalya, P.L., Mabula, J.B., Ngayomela, I.H., Kanumba, E.S., Chandika, A.B., Giiti, G., Mawala, B., Balumuka, D.D. (2010) Motorcycle injuries as an emerging public health problem in Mwanza City, north-western Tanzania. Tanzania Journal of Health Research 12, 214-221.

Chang, H-L. \& Yeh, T-H. (2007) Motorcyclist accident involvement by age, gender, and risky behaviours in Taipei, Taiwan. Transportation Research Part F 10, 109-122

Corad, P., Bradshaw, Y., Lamsudin, R., Kasniayh, N., Costello, C. (1996) Helmets, injuries and cultural definitions: motorcycle injuries in urban Indonesia. Accident Analysis and Prevention 28, 193-200.

Dee, T. (2009) Motorcycle helmets and traffic safety. Journal of Health Economics 28, 398-412.

Galukande, M., Jombwe, J., Fualal, J. \& Gakwaya, A. (2009) Boda-boda injuries a health problem and a Burden of Disease in Uganda: A tertiary Hospital survey. East and Central African Journal of Surgery 14, 33-37.

Grima, G., Aguinaga, I., Anguinaga, F. (1995) Helmet use by drivers and passengers of motorcycle in Pamplona (Spain). European Journal of Epidemiology 11, 87-89.

Hung, D., Stevenson, M. \& Ivers, R. (2008) Barriers to, and factors associated, with observed motorcycle helmet use in Vietnam. Accident Analysis and Prevention, 40, 1627-1633.

Iribhogbe, P.E. \& Odai, E.D. (2009) Driver-related risk factors in commercial motorcycle (okada) crashes in Benin City, Nigeria. Prehospital and Disaster Medicine 24, 356-359.

Jha, N., Srinivasa, D.K., Roy, G. \& Jagdish, S. (2003) Injury pattern among road traffic accident cases: a study from South India. Indian Journal of Community Medicine 28, 85-90.

Julian, S., Cooke, C. \& Sprivuris, P. (2002) Most head injury related motorcycle crash deaths are related to poor riding practices. Emergency Medicine 14, 58-61.

Keng, S. (2005) Helmet use and motorcycle fatalities in Taiwan. Accident Analysis and Prevention 31, 349-355.

Lin, M., Chang, S-H., Huang, W., Hwang H-F. \& Pai L. (2003) Factors associated with severity of motorcycle injuries among young adult riders. Annals of Emergency Medicine 41, 783-791. 
Liu, B., Ivers, R., Norton, R., Blows, S. \& Lo, S.K. (2008) Helmets for preventing injury in motorcycle riders. Cochrane Database Systematic Review, CDo04333.

Museru, L.M. \& Leshabari, M.T. (2002) Road traffic accidents in Tanzania: A 10-year epidemiological appraisal. East and Central African Journal of Surgery 7, 23-26.

Nantulya, V.M. \& Reich, M.R (2002) The neglected epidemic: road traffic injuries in developing countries. British Medical Journal 324, 1139-1142.

Nwadiaro, H.C., Ekwe, K.K., Akpayak, I.C. \& Shitta, H. (2011) Motorcycle injuries in north-central Nigeria. Nigerian Journal of Clinical Practice 14, 186-189.

Odelowo, E.O.O. (1994) Pattern of trauma resulting from motorcycle accidents in Nigerians: a two year prospective study. African Journal of Medicine and Medical Sciences 23, 109-112.

Offner, P.J., Rivara, F.P. \& Maier, R.V. (1992) The impact of motorcycle helmet use. Journal of Trauma 32, 636-641.

Oluwadiya, K.S., Oginni, I.M., Olasinde, A.A., Odu, O.O., Olakulehin, O.A. \& Olatoke, S.A. (2004) Motorcycle limb injuries in a developing country. West African Journal of Medicine 23, 4246.

Peden, M. (2004) World Report on Road Traffic Injury Prevention-Summary. World Health Organization, Geneva.

Rowland, J., Rivara, F., Salzberg, P., Soderberg, R., Maier, R. \& Koepsell, T. (1996) Motorcycle helmet use and injury outcome and hospitalization costs from crashes in Washington State. American Journal of Public Health 86, 41-45.

Seleye-Fubara, D., Ekere, A.U. (2003) Vehicular road deaths in the Niger Delta. Orient Journal of Medicine 15, 41-44.

Solagberu, B.A., Ofoegbu, C.K.P., Nasir, A.A., Ogundipe, O.K., Adekanye, A.O. \& Abdur-Rahman, L.O. (2006) Motorcycle injuries in a developing country and the vulnerability of riders, passengers, and pedestrians. Injury Prevention 12, 266-268.

Sood, S. (1988) Survey of factors influencing injury among riders involved in motorized twowheeler accidents in India: a prospective study of 302 cases. Journal of Trauma 28, 530534 .

WHO (2006) Helmets: A Road Safety Manual for Decision-makers and Practitioners. World Health Organization, Geneva, Switzerland. 